\title{
Functional outcome of midshaft clavicle fractures treated with a pre-contoured locking plate: A prospective study
}

\author{
Pramod B Itagi ${ }^{1}$, Akash Kalaskar,** \\ ${ }^{1}$ Professor \& HOD, ${ }^{2}$ Resident, Dept. of Orthopaedics, M R Medical College, Kalaburagi, Karnataka, India
}

*Corresponding Author:

Email: iamakashkalaskar@gmail.com

\begin{abstract}
Introduction: Traditionally, displaced mid clavicular fractures have been treated with conservative methods. Conservative methods like strapping gives mediocre results. The present study has been undertaken to study the role of pre-contoured locking compression plate in the fixation of mid-third clavicle fractures.

Materials and Methods: 40 patients with mid-shaft clavicle fractures (Robinson 2B) with displacement $>2 \mathrm{cms}$ were treated with open reduction and internal fixation with a pre-contoured locking plate and functional outcome through 3 months was assessed.

Results: Union was seen radiologically in all the fractures by 10-12 weeks. The average Constant-Murley score at 3 months was 93.5. None of our patients had complications like malunion, wound dehiscence, hardware irritation, early mechanical failure, incisional numbness, infections and neurovascular damage. There were 4 patients with hardware prominence, 2 cases of hypertrophic scar formation, 2 patients with delayed union at 18 weeks and 1 case of plate loosening.

Conclusions: Pre-contoured locking plate fixation in displaced fractures of clavicle allows earlier return to a pain free functional shoulder.
\end{abstract}

Keywords: Clavicle fracture, Middle third, Pre-contoured locking plate.

\section{Introduction}

The clavicle is one of the most frequently fractured bones in the body, the fracture most often resulting from a direct blow or a fall on an outstretched arm. They are common injuries in young, active individuals, especially those who participate in activities or sports where high-speed falls (bicycling, motorcycles) or violent collisions (football, hockey) are frequent, and they account for approximately $2.6 \%$ of all fractures. ${ }^{1}$ The majority of clavicular fractures (80\% to 85\%) occur in the midshaft of the bone, where the typical compressive forces applied to the shoulder and the narrow cross section of the bone combine and result in bony failure. ${ }^{1}$ Patients are typically men in their fifth decade and the usual mechanism of injury was from a motor vehicle accident. ${ }^{1}$ There is also a relatively high associated mortality from concomitant head and chest injuries. While undisplaced fractures are predominately treated by conservative methods, fractures with displacement more than $2 \mathrm{~cm}$ when treated by nonoperative methods have higher rate of non-union, poor clinical outcome and decrease in shoulder strength and endurance. $^{2,3}$ In addition, a recent meta-analysis by McKee et al. of six randomized clinical trials of operative versus nonoperative care for displaced midshaft clavicle fractures demonstrated a reduction of non-union and symptomatic malunion from 46/200 cases $(23 \%)$ in the nonoperative group to $3 / 212$ cases $(1.4 \%)$ in the operative group. ${ }^{4}$ Nonoperative methods have higher rate of non-union, poor clinical outcome and decrease in shoulder strength and endurance. ${ }^{2,5}$ When the fractures are treated with a rigid fixation, mobility at the fracture site decreases which leads to a faster union and early return to daily activities. In addition, the plate is contoured to the shape of the clavicle with different plates for the left and right side. This reduces the operative time and irritation of the skin.

We wanted to see the functional outcome of the patients treated with a pre-contoured plate for midshaft clavicle fracture.

\section{Materials and Methods}

Forty cases of middle third fractures of clavicle (Robinson type 2B1 and 2B2) admitted to our hospital from December 2015 to April 2017 were included in the study.

Inclusion Criteria: Age 18-55 years, Displacement $>2 \mathrm{cms}$, Shortening $>2 \mathrm{cms}$

Exclusion Criteria: Open fractures, associated head injury, neurovascular injury to the ipsilateral limb

Surgical Technique: Under general anaesthesia, the patient was placed in supine position with a folded towel placed under the affected shoulder. The ipsilateral arm was draped to allow intra-operative mobilization. An incision was taken centered over the fracture site and fracture was exposed. Periosteum from the inferior surface was elevated which allowed us to place a protective instrument during drilling. The fracture was reduced with the help of bone holding forceps. Interfragmentary screw was used in oblique fractures and to fix butterfly fragments. Pre-contoured plate of the respective side and adequate length was applied on the superior surface of the clavicle. Atleast 3 screws on either side of the fracture were applied. In cases where 
there was severe communition, a no. 1 vicryl was passed around the plate and the bone such that all fragments are held together. The wound was closed in two layers.

Post-operative Protocol: IV Antibiotics were given for 5 days followed by 7 days of oral antibiotics. Wound was inspected at post-op day 2 and 5. Sutures were removed at $12^{\text {th }}$ post op day. Patients were given an arm pouch for 3 weeks after which pendulum exercises were started in arm pouch. Patients were advised not to indulge in vigorous activities. X-rays were taken at post-op, 6 weeks and 3 months. At 6 weeks the arm pouch was discontinued and full range of motion at the shoulder was started. Functional outcomes were graded based on Constant and Murley scoring for shoulder.

\section{Results}

Out of 40 patients treated, $32(80 \%)$ were male patients and the rest female. The mechanism of injury was road traffic accident in 28 patients (70\%), simple fall on shoulder in 8 patients $(20 \%)$ and fall on outstretched hand in $4(10 \%)$ patients. The average age of the patient was 35.25 years. There were 24 patients $(60 \%)$ with left sided fracture and 16 patients $(40 \%)$ with right sided fracture. $80 \%$ were Robinson type $2 \mathrm{~B} 1$ fracture and the rest were Robinson 2B2 fractures. Majority $(80 \%)$ of the patients were operated in the first week of injury.

The fracture was considered to be united clinically when there was no tenderness at the fracture site and radiologically when there was callus around the fracture. In 36 patients (90\%), the fracture united before 12 weeks whereas in 2 patients, union was seen at 14 weeks. In two other patients, union occurred at 18 weeks. It was mainly due to large butterfly fragments and communition. The average time to union was 10.5 weeks.

The overall incidence of minor complications was $22.5 \%$ but no major complications occurred. In 4 cases, plate prominence was seen, 2 of which went on to get implant removal after the fracture united. 2 patients had delayed union and in one patient $(2.5 \%)$ plate loosening was seen but the fracture eventually united and implant was removed. In 2 other cases, hypertrophic skin scar was observed. There was no case of neurovascular injury, plate breakage, non-union, infection or lung injury in our patients.

Two patients had rib fractures which was managed conservatively with a rib brace. One other patient had ipsilateral femur fracture which was fixed with an intramedullary interlocking nail. One more patient had contralateral distal radius fracture which was managed by casting.

The final functional outcome was assessed by the Constant and Murley shoulder scoring. 32 patients $(80 \%)$ had excellent outcomes, 6 cases $(15 \%)$ had good outcomes and 2 patients had fair outcomes (5\%). The average Constant Murley scores was 93.5.
Table 1: Shows classification of fractures according to robinson classification

\begin{tabular}{|l|c|c|}
\hline Robinson Classification & 2B1 & 2B2 \\
\hline Number of cases & 32 & 8 \\
\hline Percentage (of 40 cases) & $80 \%$ & $20 \%$ \\
\hline
\end{tabular}

\section{Graph: Showing incidence of complications}
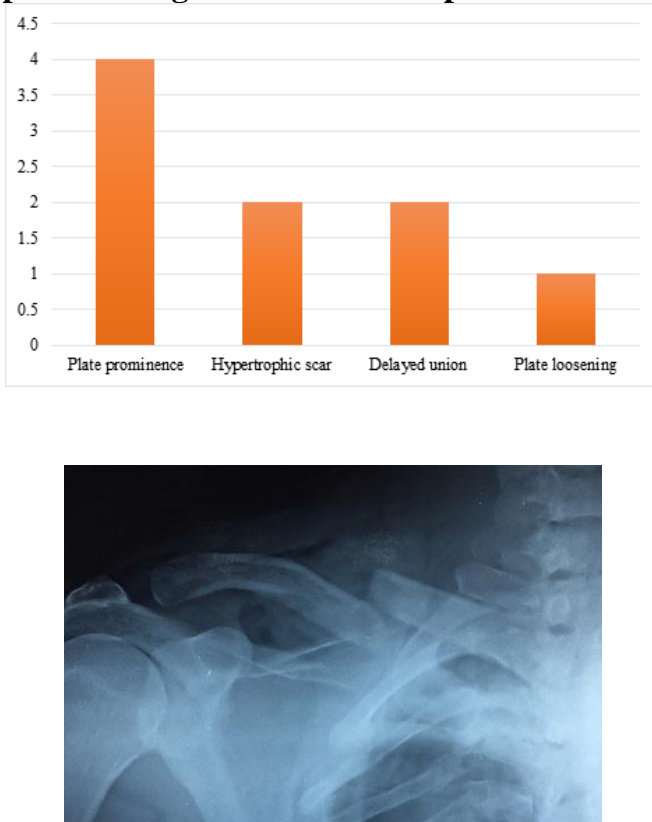

Fig. 1: Pre-operative Radiograph

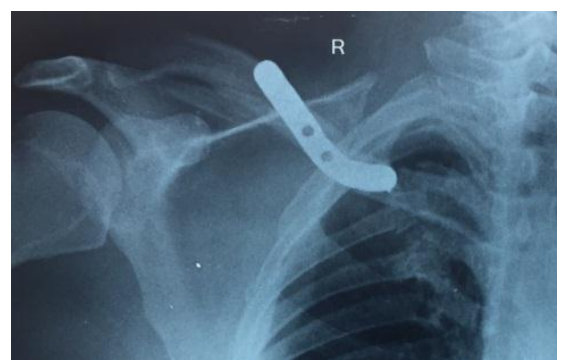

Fig. 2: Immediate post-operative radiograph

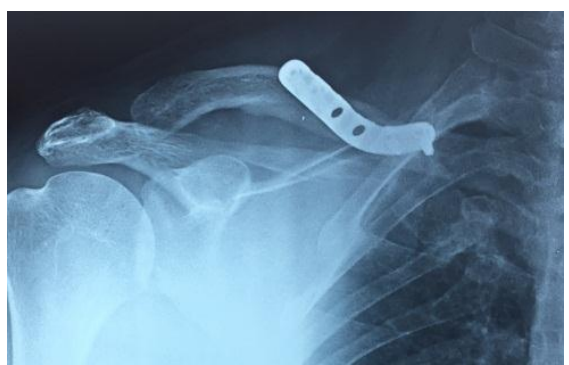

Fig. 1: X-ray showing union at final follow up 


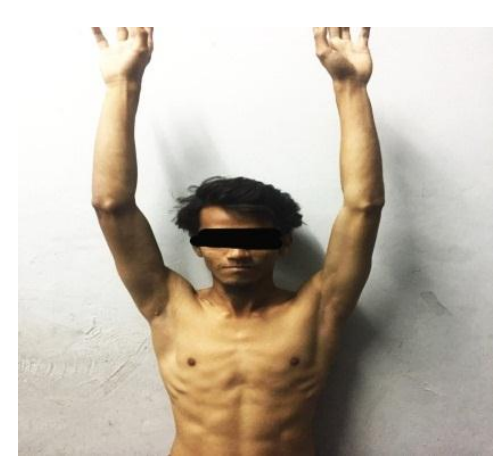

Fig. 4: Shows full abduction at 10 weeks

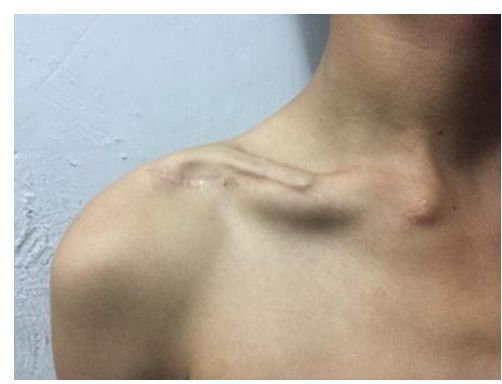

Fig. 5: Showing plate prominence

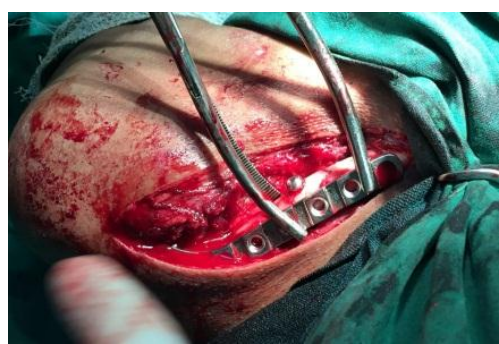

Fig. 6: Intra-operative photograph showing placement of plate on the superior surface of clavicle

\section{Discussion}

Clavicle is an S-shaped bone with a tubular cross section medially and flat laterally. The middle third is the weakest link in the bone because it lacks ligamentous and muscular supports. There are different methods of management of clavicle midshaft fracture.

Micheal Zlowodski et al conducted a meta-analysis of 2144 fractures. It was shown that a relative risk reduction of $57 \%$ for nonunion can be achieved when using a plate compared with nonoperative treatment (86\% for displaced fractures). ${ }^{6}$ In addition Patient based outcome scores in some studies have been published stating that malunion with shortening after midshaft fractures may lead to symptoms such as pain, loss of strength, rapid fatigability, paraesthesia of the arm and hand and problems with sleeping on back as well as cosmetic complaints. ${ }^{2,5,7,8}$

The procedures using $\mathrm{K}$ wire fixation, Steinman pin fixation result in low resistance to torque, carry risks of pin loosening and infection, and require a longterm fixation period. ${ }^{9,10}$ Plate osteosynthesis gives better torque resistance and obtaining anatomical reduction and compression of the fracture site, but the plates have to be bent to achieve the shape of clavicle and the fixation was difficult in communited fractures. ${ }^{9,10}$

New generation plates like pre- contoured anatomical plates are light weight, thin and give good anatomical reduction, tolerates multidirectional stress, torque and because of its locking property it gives rigid fixation in the old age osteoporotic patients. ${ }^{9,10}$

Care needs to be taken to avoid injury to subclavian nerves, vessel bundle as this may happen while drilling the opposite cortex. The only problem with this technique is the scar it leaves behind which is not preferable by most women. We had two cases of hypertrophic scar formation, 4 patients with plate prominence. Plate prominence can be avoided by antero-inferior plating, especially in lean patients. In a study conducted by Formaini $\mathrm{N}$ et al, patient-reported implant prominence was nearly double in patients with a superior plate compared with antero-inferior plate (54\% vs $29 \%$, respectively; $\mathrm{P}=.04$ ) at final follow up. ${ }^{11}$ In this study we used pre-contoured LCPs in all the patients and we experienced few complications like hardware prominence and one case of plate loosening. No cases of pain, non- union or functional disabilities were observed.

Advantages of pre-contoured anatomical LCP: Rigid fixation by locking of screws with the plate which acts as internally fixed external fixator functions as a single unit, reducing chances of screw backout or plate loosening. As these are limited contact plates, there is preservation of blood supply due to minimal surface contact of plate with bone and because of the locking property excess tightening is not possible and in return that decreases the compression of plate on the bone which helps in preservation of blood supply. ${ }^{12}$

Because of the locking property of the plate and screw it is not necessary that the tip of the screw should pass the opposite cortex but care should be taken during drilling. Decreased periosteal stripping results in rapid union. $^{12}$ Use of pre-contoured anatomical plate also decreases the procedure timing as it is pre-bent according to the shape of the clavicle.

Darren et al in a biomechanical study compared four different techniques of fixation of mid-third clavicle fractures viz. $3.5 \mathrm{~mm}$ Recon plates, $3.5 \mathrm{~mm} \mathrm{LC}$ DCP, $3.5 \mathrm{~mm}$ LCP and $4.5 \mathrm{~mm}$ Rockwood pin and found that locking plate have stiffer constructs. ${ }^{13}$ Thus, pre-contoured anatomical locking compression plate gives rigid fixation with good anatomical reduction and can be used as alternative to previously used plates.

\section{Conclusions}

Pre-contoured locking compression plate gives immediate relief of pain. They provide a rigid construct with excellent torsion stiffness. This leads to a faster 
union time and facilitates early return to function, thus avoiding shoulder stiffness. Therefore, the functional outcomes are better. Only drawback is the operative scar and prominence of plate in lean patients.

\section{Conflicts of Interest}

There are no conflicts of interest, none declared.

\section{References}

1. Rockwood CA, Bucholz RW, Court-Brown CM, Heckman JD, Tornetta P. Rockwood and Green's Fractures in Adults. Lippincott Williams \& Wilkins;2010.2059 p.

2. Hill JM, McGuire MH, Crosby LA. Closed treatment of displaced middle-third fractures of the clavicle gives poor results. J Bone Jt Surg Br. 1997;79(4):537-538.

3. Hundekar BB. Internal fixation of displaced middle third fractures of clavicle with precontoured locking plate. $\mathbf{J}$ Orthop. 2013;10(2):79-85.

4. McKee RC, Whelan DB, Schemitsch EH, McKee MD. Operative versus nonoperative care of displaced midshaft clavicular fractures: a meta-analysis of randomized clinical trials. JBJS. 2012;94(8):675-684.

5. McKee MD, Pedersen EM, Jones C, Stephen DJ, Kreder HJ, Schemitsch EH, et al. Deficits following nonoperative treatment of displaced midshaft clavicular fractures. JBJS. 2006;88(1):35-40.

6. Zlowodzki M, Zelle BA, Cole PA, Jeray K, McKee MD. Treatment of acute midshaft clavicle fractures: systematic review of 2144 fractures: on behalf of the EvidenceBased Orthopaedic Trauma Working Group. J Orthop Trauma. 2005;19(7):504-507.

7. Ledger M, Leeks N, Ackland T, Wang A. Short malunions of the clavicle: an anatomic and functional study. J Shoulder Elbow Surg. 2005;14(4):349-354.

8. Rosenberg N, Neumann L, Wallace AW. Functional outcome of surgical treatment of symptomatic nonunion and malunion of midshaft clavicle fractures. J Shoulder Elbow Surg. 2007;16(5):510-513.

9. CHARLES S NEER I. 5 Fractures of the distal third of the clavicle. Clin Orthop. 1968;58:43-50.

10. Post M. Current concepts in the treatment of fractures of the clavicle. Clin Orthop. 1989;(245):89-101.

11. Formaini N, Taylor BC, Backes J, Bramwell TJ. Superior versus anteroinferior plating of clavicle fractures. Orthopedics. 2013;36(7):e898-e904.

12. Hathiwale MI, Sasnur PA, Tapadar MJI. A prospective study on operative management of displaced fracture of midshaft of clavicle. Int J Res Orthop. 2017;3(3):619622.

13. Drosdowech DS, Manwell SE, Ferreira LM, Goel DP, Faber KJ, Johnson JA. Biomechanical analysis of fixation of middle third fractures of the clavicle. J Orthop Trauma. 2011;25(1):39-43. 